\title{
Prospective, double-blind, randomized controlled trial of electrophysiologically guided femoral nerve block in total knee arthroplasty
}

This article was published in the following Dove Press journal:

Therapeutics and Clinical Risk Management

7 March 2013

Number of times this article has been viewed

\section{Yoon Seok Youm' \\ Sung Do Cho' \\ Chang Ho Hwang ${ }^{2}$}

'Department of Orthopedic Surgery, ${ }^{2}$ Department of Physical Medicine and Rehabilitation, Ulsan University Hospital, University of Ulsan College of Medicine, Ulsan, Republic of Korea
Correspondence: Chang Ho Hwang Department of Physical Medicine and Rehabilitation, Ulsan University Hospital, University of Ulsan College of Medicine, 290-3 Jeonha-dong, Dong-gu, Ulsan 682-714, Republic of Korea

Tel +8252 2507210

Fax +82 522507211

Email chhwang1220ciba@yahoo.co.kr
Background: The purpose of this study was to compare electrophysiologically guided and traditional nerve stimulator analgesia femoral nerve block after total knee arthroplasty.

Methods: Patients scheduled for unilateral total knee arthroplasty were randomized to electrophysiologically guided or traditional nerve stimulator analgesia by pre-emptive single injection femoral nerve block with corresponding assistance. We assessed pain scores using a visual analog scale (VAS, $0=$ no pain, $100=$ the worst pain) and the volumes of morphine consumed at 4, 24, 48, and 72 hours after total knee arthroplasty.

Results: Of the 60 patients enrolled, eight withdrew from the study. The remaining 52 patients were randomized to the electrophysiologically guided group $(n=27)$ or traditional nerve stimulator analgesia $(n=25)$ group. Four hours after total knee arthroplasty, VAS scores were significantly lower in the electrophysiologically guided group than in the traditional nerve stimulator group at rest $(4.8 \pm 1.4$ versus $5.9 \pm 0.8, P<0.01)$ and while moving $(6.2 \pm 1.1$ versus $6.9 \pm 0.9, P<0.01)$. The total volumes of morphine injected at 24,48 , and 72 hours were significantly decreased in the electrophysiologically guided group $(P<0.05$ each). Variable $\times$ time interaction of VAS was significant in the electrophysiologically guided group $(P<0.05)$, with each VAS score at 24,48 , and 72 hours being significantly lower than the baseline score $(P<0.05)$. VAS scores at every time point were significantly lower in the electrophysiologically group guided than in the traditional nerve stimulator group $(P<0.05)$

Conclusion: Electrophysiologically guided single injection femoral nerve block may provide better postoperative analgesia and a greater reduction in the demand for pain killers than femoral nerve block using traditional nerve stimulator analgesia.

Keywords: femoral nerve, nerve block, electrophysiologic concepts, arthroplasty, knee, ropivacaine

\section{Introduction}

Poorly controlled pain after total knee arthroplasty may hinder early rehabilitation. Although continuous femoral nerve block is considered the ideal method of analgesia after total knee arthroplasty, ${ }^{1}$ it may have clinical disadvantages, including an increased risk of infection and reduced ambulation. The incidence of serious falls related to continuous femoral nerve block is increased within the four days postoperatively. ${ }^{2,3}$ Single injection femoral nerve block is easier and less costly to perform than continuous femoral nerve block. A recent meta-analysis showed that although both single injection and continuous femoral nerve block were superior to patient-controlled analgesia, the two former methods require further comparison. ${ }^{4}$ 
Given that the degree of neural blockage is proportional to the amount of local anesthetic, the anesthetic should be injected as close to the nerve as possible. Although femoral nerve block has been performed using a traditional nerve stimulator, the patellar motor response is not sufficiently reliable to quantify the degree of stimulation, except by phonomyography. ${ }^{5}$ Electrophysiologically guided suprascapular nerve block has been shown to be more effective than traditional suprascapular nerve block in relieving shoulder pain in patients with adhesive capsulitis. ${ }^{6}$

We hypothesized that electrophysiologic guidance would more accurately localize the target nerve than would a traditional nerve stimulator. We therefore compared the effects on postoperative analgesia of electrophysiologically guided femoral nerve block with those of femoral nerve block using a traditional nerve stimulator. To our knowledge, this is the first prospective, double-blinded, randomized, positive-controlled clinical trial evaluating electrophysiologic guidance for femoral nerve block in total knee arthroplasty.

\section{Materials and methods}

This prospective, randomized, double-blinded, parallelgroup, positive-controlled clinical trial was performed in an inpatient clinic in the department of orthopedic surgery at a general hospital in Asia from July 2006 to October 2008.

Patients scheduled for elective classic unilateral total knee arthroplasty were included. We excluded patients weighing $<50 \mathrm{~kg}$ or $>100 \mathrm{~kg}$, those with a coagulation or neurologic disease, those who were hypersensitive to local anesthetics, those not classified as American Society of Anesthesiologists (ASA) physical status I-III, and those unable to understand the pain scales or use patient-controlled analgesia. Prior to total knee arthroplasty, patients were randomized 1:1 to the electrophysiologically guided or traditional nerve stimulator groups using a random assignment generator with sequentially numbered containers (Wichmann-Hill random number generator, randomization@ com). The protocol was approved by our institutional review board (approval number UUH-06-11), and all patients provided their written informed consent.

Shortly before the surgery, each patient was aseptically draped, with subsequent topical anesthesia using $1 \mathrm{~mL}$ of $1 \%$ lidocaine. Patients were injected with a single dose of ropivacaine for femoral nerve block according to the landmarks of Winnie et al. ${ }^{7}$ No sedative was administered.

The medical equipment (Medelec Synergy, Vickers Medical, Surrey, UK) used comprised three parts, the first being to stimulate the nerve, the second being to take up the electrical signal, and the third being to display the signal on the screen as a graph.

Patients in the electrophysiologically guided group were placed in the supine position, and the femoral artery was palpated below the inguinal ligament. A $7 \mathrm{~mm}, 22$ gauge, Teflon-coated, electrically conductive, double-lumen, beveled cannula (Myojet disposable hypodermic needle electrode, TECA Accessories, New York, NY, USA) was inserted just lateral to the femoral artery and into the iliopsoas muscle. This cannula enabled both femoral nerve stimulation and injection of local anesthetic. A recording electrode was placed on the skin above the motor point of the vastus medialis showing the maximum electrical signal of muscle contraction. The femoral nerve was located by nerve stimulation at a frequency of $1 \mathrm{~Hz}$, a duration of $0.2 \mathrm{msec}$, and an intensity of $5 \mathrm{~mA}$, while monitoring the change in amplitude of the electrical signal. Once the electrical signal was found, the stimulation intensity was reduced stepwise by moving the cannula back and forth and side to side until the highest amplitude could be induced by a stimulation intensity $<0.5 \mathrm{~mA}$. After the aspiration test showed no evidence of blood, $10 \mathrm{~mL}$ of $0.375 \%$ ropivacaine was injected. To confirm the correct level of analgesia, we monitored the disappearance of the electrical signal by stimulation with an intensity of $5 \mathrm{~mA}$ shortly after injection and by assessment of the integumentary sensation in the dermatome of the femoral nerve by pinprick tests (Figure 1A and B).

Patients in the traditional nerve stimulator group received a single injection femoral nerve block as described for the electrophysiologically guided group, using the same medical equipment, nerve stimulation of equal parameters, and downward adjustment of intensity below $0.5 \mathrm{~mA}$, while monitoring brisk cephalic patellar tilting, a marker for contractions of the quadriceps femoris. Loss of sensation was determined upon completion of femoral nerve block.

General anesthesia was induced by injection of alfentanil $15 \mu \mathrm{g} / \mathrm{kg}$, propofol $2-2.5 \mathrm{mg} / \mathrm{kg}$, and rocuronium $0.6-0.8$ $\mathrm{mg} / \mathrm{kg}$, maintained with $40 \%-60 \%$ oxygen-nitrous oxide and $1 \%-2 \%$ sevoflurane, and reversed with neostigmine $40 \mu \mathrm{g} / \mathrm{kg}$ and glycopyrrolate $7 \mu \mathrm{g} / \mathrm{kg}$. Alfentanil $0.25 \mathrm{mg}$ was injected intravenously if mean arterial pressure or heart rate was $>125 \%$ of preoperative values.

Supplemental postoperative analgesia after total knee replacement was standardized. ${ }^{8}$ The morphine dose was half that recorded for Caucasians, due to the smaller body weight and volume of Asian. Pain was controlled by intravenous patient-controlled analgesia morphine in $1 \mathrm{mg}$ increments, with a 5-minute lockout time up to a total limit of $4 \mathrm{mg}$. 

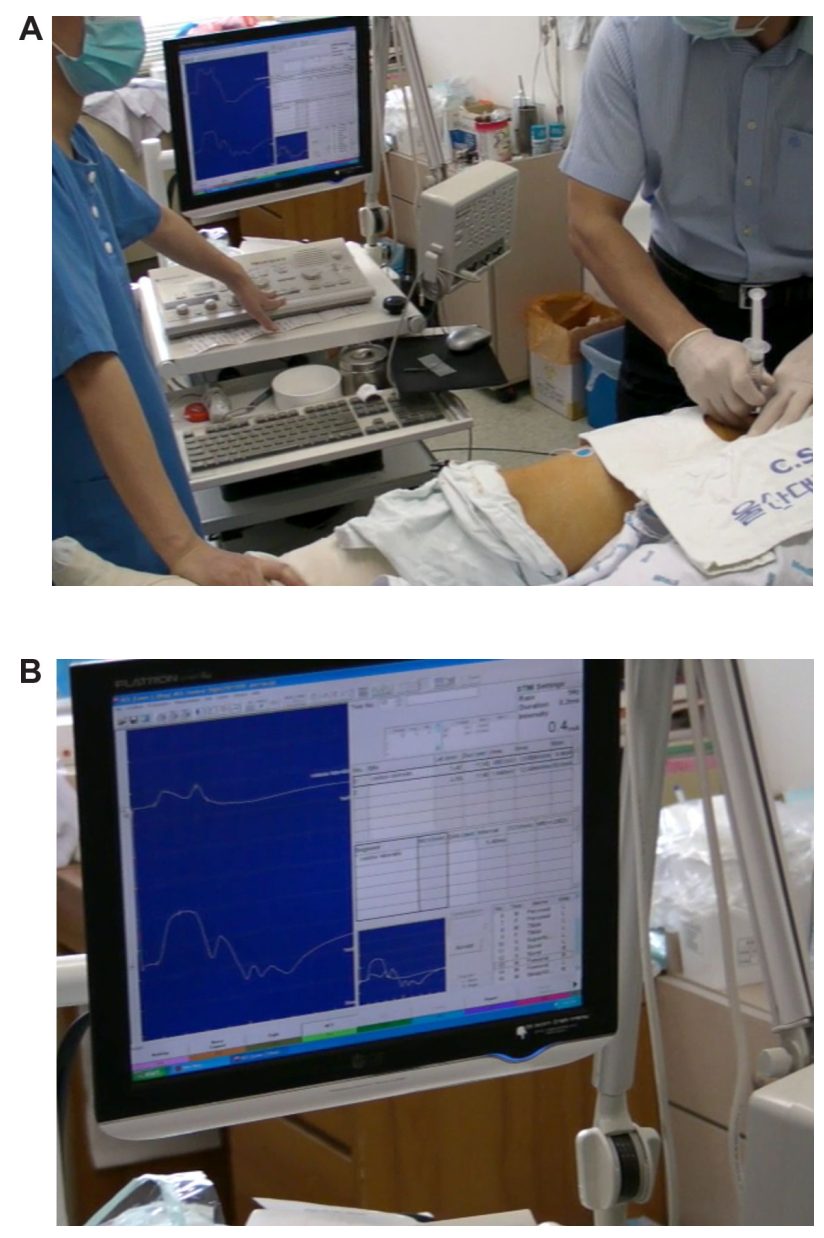

Figure I (A and B) Electrophysiologically guided femoral nerve block.

Pain while at rest and moving was assessed using a visual analog scale (VAS, $0=$ no pain, $100=$ the worst pain) and postoperative pain score $(0=$ no pain; $1=$ moderate pain only while moving; $2=$ moderate pain while resting or

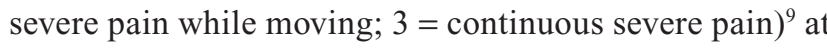
hours 4, 24, 48, and 72 after the end of the surgery. If the VAS pain score exceeded 4 after $4 \mathrm{mg}$ of morphine, $1 \mathrm{mg}$ increments were allowed until the score was reduced to 4 . Each patient was administered two tablets of acetaminophen $250 \mathrm{mg}$ plus codeine $10 \mathrm{mg}$ one hour after surgery, followed by one tablet three times per day. The number of intravenous patient-controlled analgesia doses and the total amount of morphine every 3 days were recorded. Nausea and vomiting were recorded only if an antiemetic drug was used. Major side effects of ropivacaine, including convulsions and hypotension, were recorded, ${ }^{10}$ as were urinary retention needing catheterization or prolonged muscle paralysis.

Patients in both groups started an identical physiotherapy regimen the day after surgery. All staff members, including the surgical teams, nurses, assessors, and physiotherapists, were blinded to patient assignment.

\section{Statistical analysis}

All statistical analyses were performed using SPSS version 19.0 for Windows (SPSS Inc, Chicago, IL, USA). An alpha level $<0.05$ was considered to be statistically significant. The power value was determined to be $80 \%$, with an assumed dropout rate of $20 \%$. A preliminary study found a standardized difference of 0.8 . Using Lehr's formula, we calculated that 60 patients would be required. Demographic factors were compared using the Chi-square and two-sample Student's $t$-tests. Variable $\times$ time and variable $\times$ group interactions of the pain scales were evaluated by repeated-measures analysis of variance. Total amounts of morphine consumed and pain scores were evaluated by two-sample paired $t$-tests or Student's $t$-tests.

\section{Results}

Of the 97 patients screened, 37 were excluded. Sixty patients were recruited from July 2006 to September 2008, with 30 assigned to each group. Three patients in the electrophysiologically guided group were lost to follow-up, one due to no femoral nerve block and two due to poor standardization of postoperative analgesia, as were five patients in the traditional nerve stimulator group, two due to no femoral nerve block and three due to poor standardization of postoperative analgesia. Therefore, our per-protocol analysis included 27 patients in the electrophysiologically guided group and 25 in the traditional nerve stimulator group (Figure 2).

Pain scores and morphine consumption were assessed in the recovery room and the general ward at 4, 24, 48, and 72 hours after the end of surgery. There were no failures of femoral nerve block, except for one patient with severe anxiety.

The two groups were well matched in terms of demographics and preoperative pain scores $(P=0.23-0.97$, Table 1$)$. There was no regulation of the gender ratio recruited.

All patients in both groups showed improvement in pain scores 4 hours after total knee arthroplasty $(P<0.01)$. Postoperative VAS score was significantly lower in the electrophysiologically guided group than in the traditional nerve stimulator group at 4 hours, both while at rest $(48 \pm 1.4$ versus $59 \pm 0.8, P<0.01)$ and while moving $(62 \pm 1.1$ versus $69 \pm 0.9, P<0.01$, Table 2 ). However, postoperative pain scores at 4 hours did not differ significantly.

Variable $\times$ group interactions of VAS score did not differ significantly, indicating that constant VAS intervals were 


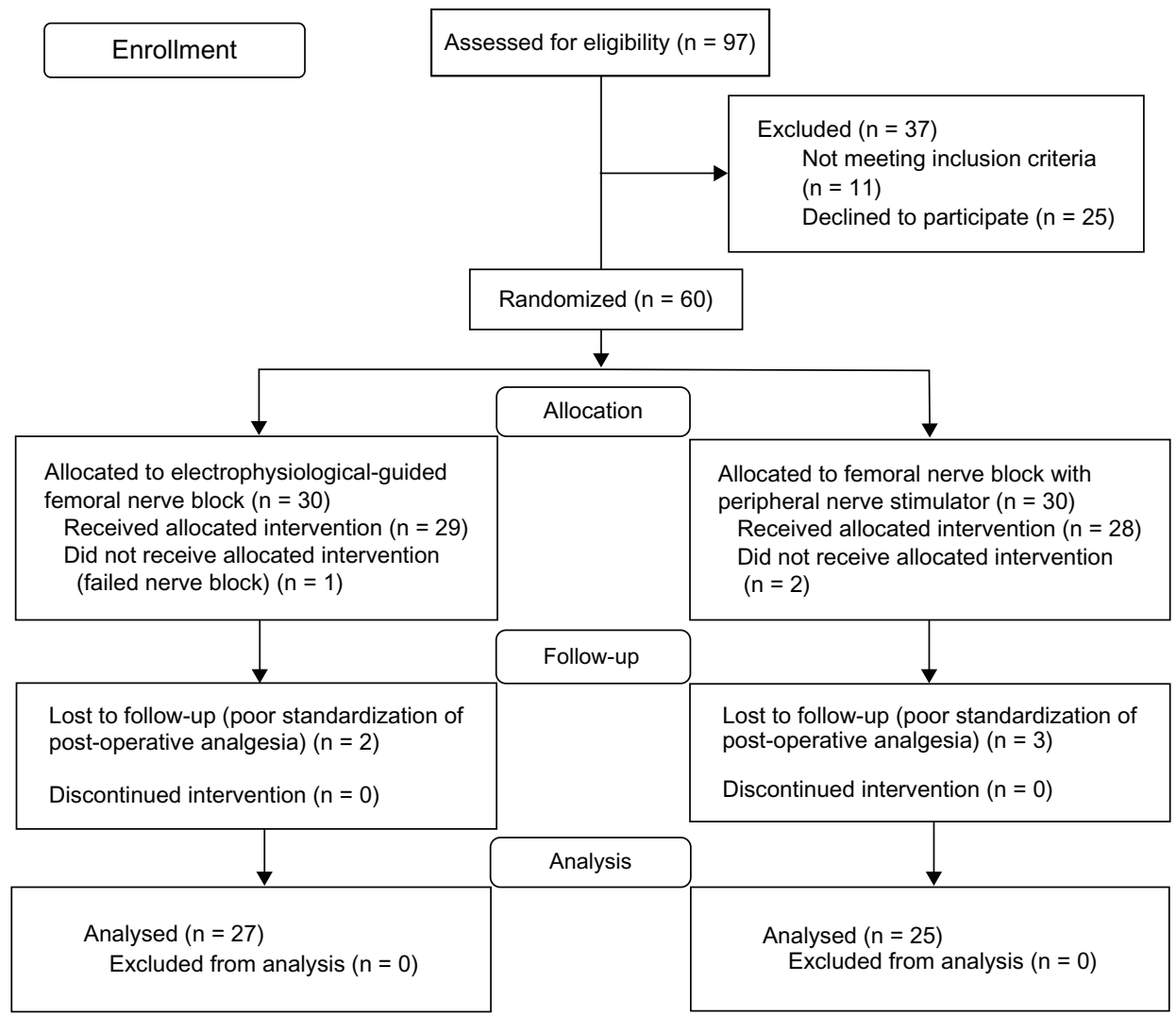

Figure 2 Participant flow.

present at each endpoint in the two groups. Variable $\times$ time interactions of VAS score differed significantly while at rest $(\mathrm{F}[2.1,102.9]=96.8, P<0.01)$ and while moving $(\mathrm{F}[2.4,118.2]=99.9, P<0.01)$, showing significant sequential changes within the groups over time. Within each group, VAS score was significantly lower at 24, 48, and 72 hours than at baseline, both while at rest and while moving $(P<0.01)$. At all four time points, ie, 4, 24, 48, and 72 hours after total knee arthroplasty, the mean VAS score was significantly lower in the electrophysiologically

Table I Demographic data

\begin{tabular}{lll}
\hline & $\begin{array}{l}\text { EG group } \\
(\mathbf{n}=\mathbf{2 7})\end{array}$ & $\begin{array}{l}\text { TNS group } \\
(\mathbf{n}=\mathbf{2 5})\end{array}$ \\
\hline Age (years) & $62.3 \pm 9.3$ & $61.8 \pm 6.3$ \\
Gender (F/M) & $25 / 2$ & $23 / 2$ \\
Weight $(\mathrm{kg})$ & $62.2 \pm 14.1$ & $64.9 \pm 14.2$ \\
Height $(\mathrm{cm})$ & $155.3 \pm 8.1$ & $152.2 \pm 10.7$ \\
ASA I/II/III & $10 / 8 / 9$ & $9 / 6 / 10$ \\
VAS while at rest & $88 \pm 7$ & $89 \pm 8$ \\
VAS while moving & $91 \pm 6$ & $92 \pm 5$ \\
\hline
\end{tabular}

Note: Values are the mean \pm standard deviation (median).

Abbreviations: ASA, American Society of Anesthesiologists physical status; VAS, visual analog score; EG, electrophysiologically guided; TNS, traditional nerve stimulator. guided group than in the traditional nerve stimulator group $(P<0.01$, Table 2, Figure 3$)$. Variable $\times$ time and variable $\times$ group interactions for postoperative pain score were not significant.

The total amounts of morphine consumed were significantly lower in the electrophysiologically guided group than in the traditional nerve stimulator group at $24(5.9 \pm 1.8 \mathrm{mg}$ versus $6.9 \pm 1.7 \mathrm{mg}, P<0.05), 48(2.9 \pm 1.6 \mathrm{mg}$ versus $3.5 \pm 2.0 \mathrm{mg}, P<0.05)$, and $72(1.9 \pm 1.6 \mathrm{mg}$ versus $3.2 \pm 1.9 \mathrm{mg}, P<0.05$ ) hours (Table 3 ).

Table 2 VAS at postoperative hours 4, 24, 48, and 72

\begin{tabular}{lcccc}
\hline & $\mathbf{4}$ hours & $\mathbf{2 4}$ hours & $\mathbf{4 8}$ hours & $\mathbf{7 2}$ hours \\
\hline $\begin{array}{l}\text { VAS at rest } \\
\text { EG guided group } \\
(\mathrm{n}=27)\end{array}$ & $48 \pm 14^{*,+}$ & $41 \pm 12^{*}$ & $32 \pm 12^{*}$ & $25 \pm 13^{*}$ \\
$\begin{array}{l}\text { TNS group } \\
(\mathrm{n}=25)\end{array}$ & $59 \pm 8$ & $50 \pm 9$ & $38 \pm 13$ & $36 \pm 12$ \\
VAS moving & & & & \\
EG group & $62 \pm 11^{*, \dagger}$ & $53 \pm 13^{*}$ & $45 \pm 13^{*}$ & $37 \pm 17^{*}$ \\
TNS group & $69 \pm 9$ & $55 \pm 9$ & $50 \pm 12$ & $48 \pm 13$ \\
\hline
\end{tabular}

Notes: Values are the mean \pm standard deviation. $* P<0.01$ in repeated-measures analysis of variance; ${ }^{\dagger} P<0.01$ in two-sample Student's $t$-test.

Abbreviations: ASA, American Society of Anesthesiologists physical status; VAS, visual analog score; EG, electrophysiologically guided; TNS, traditional nerve stimulator. 


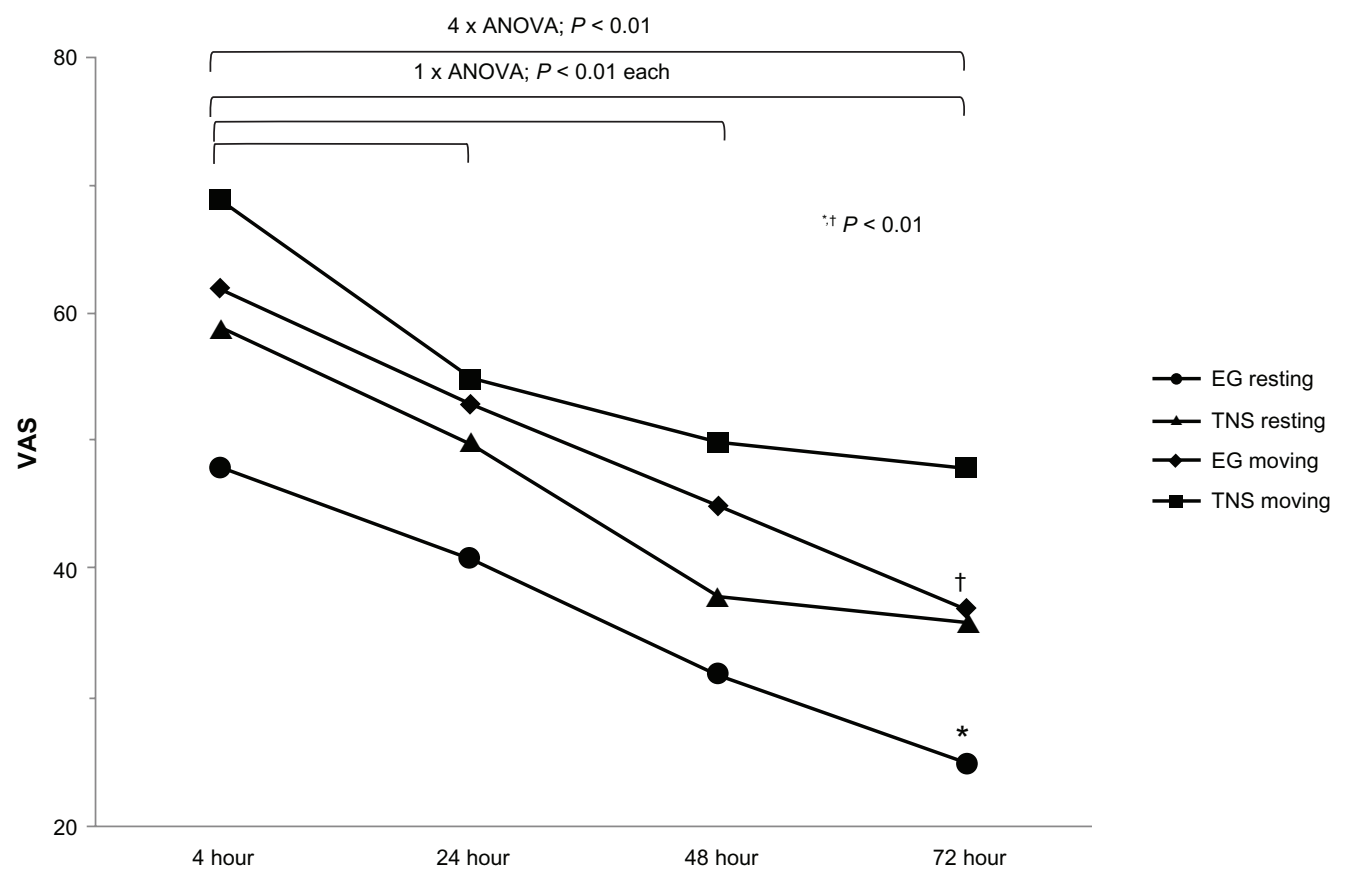

Figure 3 Mean postoperative VAS changes over 3 days.

Notes: EG resting, VAS while at rest in the EG group; TNS resting, VAS while at rest in the TNS group; EG moving, VAS while moving in the EG group; TNS moving, VAS while moving in the TNS group. Significant sequential reduction of VAS while at rest and moving in the EG group was found in repeated-measures analysis of variance $(P<0.0 \mathrm{l})$.

Abbreviations: EG, electrophysiologically guided; TNS, traditional nerve stimulator; VAS, visual analog score.

\section{Side effects}

No patient experienced any serious adverse effects, such as seizure, hypotension, urinary retention, or prolonged motor palsy. Two patients in the traditional nerve stimulator group required injections of antiemetic drugs within 24 hours.

\section{Discussion}

We have evaluated the relative effects of electrophysiologically guided and traditional nerve stimulator femoral nerve block. The major finding of this study was that electrophysiologically guided preoperative femoral nerve block reduced postoperative pain and morphine consumption more than did the traditional nerve stimulator.

Femoral nerve block has been performed by checking contractions of the quadriceps femoris or brisk patellar tilting induced by a traditional nerve stimulator. However, visual determination of muscle retraction is not ideal for quantification. Although electrodiagnostic methods have been used to evaluate neurologic disease, they have not been used previously to assess peripheral nerve blockade, although a few recent trials have described the use of an electrophysiologic approach. ${ }^{11,12}$

Electrodiagnostic studies of motor nerves rely on the magnifying effect. Stimulating one motor axon can induce activation of many adjacent muscle fibers simultaneously, depending on the innervation ratio of the target muscle. Amplified electrical impulses are recorded and displayed on a screen in the form of a graph. Electrodiagnostic methods can assist in determining the maximum amplitude of electrical signals generated by stimulation with a minimum intensity, enabling real-time quantification. ${ }^{13}$ Theoretically, electrophysiologic guidance can be superior to visual assessment in standardization, with more precise quantification, resulting in better nerve location and more accurate nerve blockage.

In patients undergoing knee arthroscopy, ultrasound guidance has been reported to reduce the minimum effective volume of anesthetic required to block the sciatic nerve when compared with a traditional nerve stimulator. ${ }^{14}$ Continuous femoral nerve block with ultrasound reduced the volumes of local anesthetics and morphine consumption, as well as postoperative pain scores, when compared with a traditional nerve stimulator. ${ }^{15}$ Thus, regardless of the type of modality, accurate localization can enhance the efficacy of nerve blockade.

The failure rate of femoral nerve block is about $5 \%,{ }^{16}$ with one important reason being anatomic variation. ${ }^{17}$ For example, anatomic variation in the femoral nerve have been reported in $35 \%$ of patients, ${ }^{18}$ with variable muscular branches or slips dividing the nerve. None of our patients experienced 
Table 3 Morphine consumption

\begin{tabular}{lll}
\hline $\begin{array}{l}\text { Cumulative amount } \\
\text { of injected morphine }\end{array}$ & $\begin{array}{l}\text { EG group } \\
(\mathbf{n}=\mathbf{2 7})\end{array}$ & $\begin{array}{l}\text { TNS group } \\
(\mathbf{n}=\mathbf{2 5})\end{array}$ \\
\hline 24 hours & $5.9 \pm 1.8 \mathrm{mg}$ & $6.9 \pm 1.7 \mathrm{mg}$ \\
48 hours & $2.9 \pm 1.6 \mathrm{mg}$ & $3.5 \pm 2.0 \mathrm{mg}$ \\
72 hours & $1.9 \pm 1.6 \mathrm{mg}$ & $3.2 \pm 1.9 \mathrm{mg}$ \\
\hline
\end{tabular}

Notes: $* P<0.05$. Values are the mean \pm standard deviation.

Abbreviations: EG, electrophysiologically guided; TNS, traditional nerve stimulator.

failure of femoral nerve block. Electrophysiologic guidance, which displays the shape of electrical impulses on a screen, has the advantage of finding nerve variations (compound motor action potential). Given that normal curves are biphasic, ${ }^{13}$ a division of the first peak into two or more peaks would suggest the presence of an anatomic variation. Thus, electrophysiologic guidance may reduce the failure rate of femoral nerve block.

The rate of femoral nerve injury due to puncture or intraneural injection has been reported to be $0.7 \%$ in 709 femoral nerve blocks. ${ }^{19}$ None of our patients experienced femoral neuritis or neuropathy. Conduction block, defined as an inhibition in the spread of an electrical current resulting in a reduction in amplitude to $80 \%-90 \%$, may be induced if stimulation with an intensity $>1.0 \mathrm{~mA}$ is performed within $2 \mathrm{~mm}$ of the epineurium in rat sciatic nerve. ${ }^{12}$ The extraneural threshold for activation ranges from 0.3 to $0.6 \mathrm{~mA}$ in humans, with an intraneural threshold of $0.3 \mathrm{~mA} .{ }^{20,21}$ However, there have been no reports to date on the range of amplitude in which conduction blocks occur in humans. It is difficult to detect a small reduction (10\%-20\%) in amplitude by observing muscle contractions or patellar movement. Electrophysiologic guidance can allow quantitative comparison of amplitude, thus avoiding injury to the nerve. Because eight patients violated the protocol, we utilized per-protocol analysis rather than intention-to-treat analysis. However, per-protocol analysis may overestimate results. ${ }^{22}$

Knee pain can originate from the distribution of obturator or lateral femoral cutaneous nerves. ${ }^{7}$ Fascia iliaca block ("3-in-1") has been developed for complete analgesia, with large volumes $(20-60 \mathrm{~mL})$ required. The volume we used $(10 \mathrm{~mL})$ may be insufficient for complete blockade of two nerves. Moreover, injection of sufficient volume may not completely block two nerves. ${ }^{23,24}$ Because we did not determine whether the obturator or lateral femoral cutaneous nerve was blocked, the result we observed may not represent the only effect of femoral nerve block. In addition, sciatica and hip joint disorders may mask the pain of osteoarthritis knee joint, and we did not exclude patients with comorbidities of the spine and hip.
The clinical effectiveness of femoral nerve block has been assessed using functional outcomes such as length of hospital stay and range of motion. ${ }^{25,26}$ However, we did not functionally evaluate our patients. Although total knee arthroplasty is performed predominantly in female patients in our country, the gender ratio of our patients may not represent that of patients undergoing total knee arthroplasty. Another limitation may be poor standardization of other factors affecting pain induction, such as duration of surgery and amount of opioid or pain killers used during surgery.

\section{Conclusion}

Electrophysiologically guided single injection femoral nerve block can provide better pain relief than traditional nerve stimulator femoral nerve block in patients undergoing total knee arthroplasty, as well as reducing the consumption of analgesics, suggesting that the electrophysiologically guided approach may prove more efficacious in localizing nerves. Except for its greater cost, the electrophysiologically guided approach may be a good alternative, especially for physicians experienced in electrodiagnosis.

\section{Acknowledgments}

Authors are grateful to Geum Mi Lee, nurse practitioner, for collection of data and coordination for scheduling and Mi Jeong Kim, medical technologist, and Dae-Sik Son and Jin Wan Seong, residents, for their valued assistance on the electrophysiological intervention.

\section{Disclosure}

The authors report no conflicts of interest in this work.

\section{References}

1. Hadzic A, Houle TT, Capdevila X, Ilfeld BM. Femoral nerve block for analgesia in patients having knee arthroplasty. Anesthesiology. 2010;113(5):1014-1015

2. Ilfeld BM, Duke KB, Donohue MC. The association between lower extremity continuous peripheral nerve blocks and patient falls after knee and hip arthroplasty. Anesth Analg. 2010;111(6):1552-1554.

3. Feibel RJ, Dervin GF, Kim PR, Beaule PE. Major complications associated with femoral nerve catheters for knee arthroplasty: a word of caution. J Arthroplasty. 2009;24(Suppl 6):132-137.

4. Paul JE, Arya A, Hurlburt L, et al. Femoral nerve block improves analgesia outcomes after total knee arthroplasty: a meta-analysis of randomized controlled trials. Anesthesiology. 2010;113(5):1144-1162.

5. Michaud G, Trager G, Deschamps S, Hemmerling TM. Monitoring neuromuscular blockade at the vastus medialis muscle using phonomyography. Can J Anaesth. 2005;52(8):795-800.

6. Karatas GK, Meray J. Suprascapular nerve block for pain relief in adhesive capsulitis: comparison of 2 different techniques. Arch Phys Med Rehabil. 2002;83(5):593-597.

7. Winnie AP, Ramamurthy S, Durrani Z. The inguinal paravascular technic of lumbar plexus anesthesia: the "3-in-1 block". Anesth Analg. 1973;52(6): 989-996. 
8. Ritchie ED, Tong D, Chung F, Norris AM, Miniaci A, Vairavanathan SD. Suprascapular nerve block for postoperative pain relief in arthroscopic shoulder surgery: a new modality? Anesth Analg. 1997;84(6): 1306-1312.

9. Singelyn FJ, Gouverneur JM. Postoperative analgesia after total hip arthroplasty: iv PCA with morphine, patient-controlled epidural analgesia, or continuous " 3 -in-1" block: a prospective evaluation by our acute pain service in more than 1,300 patients. J Clin Anesth. 1999; 11(7):550-554.

10. Simpson D, Curran MP, Oldfield V, Keating GM. Ropivacaine: a review of its use in regional anaesthesia and acute pain management. Drugs. 2005;65(18):2675-2717.

11. Fuzier R, Richez AS, Choquet O, Singelyn F. Electrical nerve localization: non-linear relationship between intensity and pulse duration to stimulate a nerve. Ann Fr Anesth Reanim. 2008;27(10):802-807. French.

12. Li J, Kong X, Gozani SN, Shi R, Borgens RB. Current-distance relationships for peripheral nerve stimulation localization. Anesth Analg 2011;112(1):236-241.

13. Wilbourn AJ. Nerve conduction studies. Types, components, abnormalities, and value in localization. Neurol Clin. 2002;20(2):305-338.

14. Danelli G, Ghisi D, Fanelli A, et al. The effects of ultrasound guidance and neurostimulation on the minimum effective anesthetic volume of mepivacaine $1.5 \%$ required to block the sciatic nerve using the subgluteal approach. Anesth Analg. 2009;109(5):1674-1678.

15. Aveline C, Le Roux A, Le Hetet H, Vautier P, Cognet F, Bonnet F. Postoperative efficacies of femoral nerve catheters sited using ultrasound combined with neurostimulation compared with neurostimulation alone for total knee arthroplasty. Eur JAnaesthesiol. 2010; 27(11):978-984.

16. Gurnaney H, Ganesh A, Cucchiaro G. The relationship between current intensity for nerve stimulation and success of peripheral nerve blocks performed in pediatric patients under general anesthesia. Anesth Analg 2007;105(6):1605-1609.
17. Kirchmair L, Lirk P, Colvin J, Mitterschiffthaler G, Moriggl B. Lumbar plexus and psoas major muscle: not always as expected. Reg Anesth Pain Med. 2008;33(2):109-114.

18. Anloague PA, Huijbregts P. Anatomical variations of the lumbar plexus: a descriptive anatomy study with proposed clinical implications. J Man Manip Ther. 2009;17(4):e107-e114.

19. Sharma S, Iorio R, Specht LM, Davies-Lepie S, Healy WL. Complications of femoral nerve block for total knee arthroplasty. Clin Orthop Relat Res. 2010;468(1):135-140.

20. Hadzic A, Vloka JD, Claudio RE, Hadzic N, Thys DM, Santos AC. Electrical nerve localization: effects of cutaneous electrode placement and duration of the stimulus on motor response. Anesthesiology. 2004;100(6):1526-1530.

21. Bigeleisen PE, Moayeri N, Groen GJ. Extraneural versus intraneural stimulation thresholds during ultrasound-guided supraclavicular block. Anesthesiology. 2009;110(6):1235-1243.

22. Melander H, Ahlqvist-Rastad J, Meijer G, Beermann B. Evidence b(i) ased medicine - selective reporting from studies sponsored by pharmaceutical industry: review of studies in new drug applications. $B M J$. 2003;326(7400):1171-1173.

23. Cauhepe C, Oliver M, Colombani R, Railhac N. The "3-in-1" block: myth or reality? Ann Fr Anesth Reanim. 1989;8(4):376-378. French.

24. Marhofer P, Nasel C, Sitzwohl C, Kapral S. Magnetic resonance imaging of the distribution of local anesthetic during the three-in-one block. Anesth Analg. 2000;90(1):119-124.

25. Singelyn FJ, Deyaert M, Joris D, Pendeville E, Gouverneur JM. Effects of intravenous patient-controlled analgesia with morphine, continuous epidural analgesia, and continuous three-in-one block on postoperative pain and knee rehabilitation after unilateral total knee arthroplasty. Anesth Analg. 1998;87(1):88-92.

26. Wang H, Boctor B, Verner J. The effect of single-injection femoral nerve block on rehabilitation and length of hospital stay after total knee replacement. Reg Anesth Pain Med. 2002;27(2):139-144.
Therapeutics and Clinical Risk Management

\section{Publish your work in this journal}

Therapeutics and Clinical Risk Management is an international, peerreviewed journal of clinical therapeutics and risk management, focusing on concise rapid reporting of clinical studies in all therapeutic areas, outcomes, safety, and programs for the effective, safe, and sustained use of medicines. This journal is indexed on PubMed Central, CAS,

\section{Dovepress}

EMBase, Scopus and the Elsevier Bibliographic databases. The manuscript management system is completely online and includes a very quick and fair peer-review system, which is all easy to use. Visit http://www.dovepress.com/testimonials.php to read real quotes from published authors. 